\title{
Sentence Embeddings in NLI with Iterative Refinement Encoders
}

\author{
Aarne Talman, Anssi Yli-Jyrä and Jörg Tiedemann \\ Department of Digital Humanities \\ University of Helsinki \\ \{aarne.talman, anssi.yli-jyra, jorg.tiedemann\}@helsinki.fi
}

\begin{abstract}
Sentence-level representations are necessary for various NLP tasks. Recurrent neural networks have proven to be very effective in learning distributed representations and can be trained efficiently on natural language inference tasks. We build on top of one such model and propose a hierarchy of BiLSTM and max pooling layers that implements an iterative refinement strategy and yields state of the art results on the SciTail dataset as well as strong results for SNLI and MultiNLI. We can show that the sentence embeddings learned in this way can be utilized in a wide variety of transfer learning tasks, outperforming InferSent on 7 out of 10 and SkipThought on 8 out of 9 SentEval sentence embedding evaluation tasks. Furthermore, our model beats the InferSent model in 8 out of 10 recently published SentEval probing tasks designed to evaluate sentence embeddings' ability to capture some of the important linguistic properties of sentences.
\end{abstract}

\section{Introduction}

Neural networks have been shown to provide a powerful tool for building representations of natural languages on multiple levels of linguistic abstraction. Perhaps the most widely used representations in natural language processing are word embeddings (Mikolov, Sutskever, Chen, Corrado, and Dean 2013, Pennington, Socher, and Manning 2014). Recently there has been a growing interest in models for sentencelevel representations using a range of different neural network architectures. Such sentence embeddings have been generated using unsupervised learning approaches (Kiros, Zhu, Salakhutdinov, Zemel, Urtasun, Torralba, and Fidler 2015, Hill, Cho, and Korhonen 2016), and supervised learning (Bowman, Gauthier, Rastogi, Gupta, Manning, and Potts 2016, Conneau, Kiela, Schwenk, Barrault, and Bordes 2017).

Supervision typically comes in the form of an underlying semantic task with labeled data to train the model. The most prominent task for that purpose is natural language inference (NLI) that tries to model the inferential relationship between two or more given sentences. In particular, given two sentences - the premise $p$ and the hypothesis $h$ - the task is to determine whether $h$ is entailed by $p$, whether the sentences are in contradiction with each other or whether there is no inferential relationship between the sentences (neutral). There are two main neural approaches 
to NLI. Sentence encoding-based models focus on building separate embeddings for the premises and the hypothesis and then combine those using a classifier (Bowman, Angeli, Potts, and Manning 2015, Bowman et al.|2016. Conneau et al.|2017). Other approaches do not treat the two sentences separately but utilize e.g. cross-sentence attention (Tay, Tuan, and Hui 2018, Chen, Zhu, Ling, Wei, Jiang, and Inkpen 2017a).

With the goal of obtaining general-purpose sentence representations in mind, we opt for the sentence encoding approach. Motivated by the success of the InferSent architecture (Conneau et al. 2017) we extend their architecture with a hierarchylike structure of bidirectional LSTM (BiLSTM) layers with max pooling. All in all, our model improves the previous state of the art for SciTail (Khot, Sabharwal, and Clark 2018) and achieves strong results for the SNLI and Multi-Genre Natural Language Inference corpus (MultiNLI; Williams, Nangia, and Bowman 2018).

In order to demonstrate the semantic abstractions achieved by our approach, we also apply our model to a number of transfer learning tasks using the SentEval testing library (Conneau et al. 2017), and show that it outperforms the InferSent model on 7 out of 10 and SkipThought (Kiros et al. 2015) on 8 out of 9 tasks, comparing to the scores reported by Conneau et al. (2017). Moreover, our model outperforms the InferSent model in 8 out of 10 recently published SentEval probing tasks designed to evaluate sentence embeddings' ability to capture some of the important linguistic properties of sentences (Conneau, Kruszewski, Lample, Barrault, and Baroni|2018). This highlights the generalization capability of the proposed model, confirming that its architecture is able to learn sentence representations with strong performance across a wide variety of different NLP tasks.

\section{Related Work}

There is a wide variety of approaches to sentence-level representations that can be used in natural language inference. Bowman et al. (2015) and Bowman et al. (2016) explore RNN and LSTM architectures, Mou, Men, Li, Xu, Zhang, Yan, and Jin (2016) convolutional neural networks and Vendrov, Kiros, Fidler, and Urtasun (2016) GRUs, to name a few. The basic idea behind these approaches is to encode the premise and hypothesis sentences separately and then combine those using a neural network classifier.

Conneau et al. (2017) explore multiple different sentence embedding architectures ranging from LSTM, BiLSTM and intra-attention to convolution neural networks and the performance of these architectures on NLI tasks. They show that, out of these models, BiLSTM with max pooling achieves the strongest results not only in NLI but also in many other NLP tasks requiring sentence level meaning representations. They also show that their model trained on NLI data achieves strong performance on various transfer learning tasks.

Although sentence embedding approaches have proven their effectiveness in NLI, there are multiple studies showing that treating the hypothesis and premise sentences together and focusing on the relationship between those sentences yields better results (Tay et al. 2018, Chen et al. 2017a). These methods are focused on the 
inference relations rather than the internal semantics of the sentences. Therefore, they do not offer similar insights about the sentence level semantics, as individual sentence embeddings do, and they cannot straightforwardly be used outside of the NLI context.

\section{Model Architecture}

Our proposed architecture follows a sentence embedding-based approach for NLI introduced by Bowman et al. (2015). The model illustrated in Figure 1 contains sentence embeddings for the two input sentences, where the output of the sentence embeddings are combined using a heuristic introduced by Mou et al. (2016), putting together the concatenation $(u, v)$, absolute element-wise difference $|u-v|$, and element-wise product $u * v$. The combined vector is then passed on to a 3 layered multi-layer perceptron (MLP) with a 3-way softmax classifier. The first two layers of the MLP both utilize dropout and a ReLU activation function.

We use a variant of ReLU called Leaky ReLU (Maas, Hannun, and Ng 2013), defined by:

$$
\text { LeakyReLU }(x)=\max (0, x)+y * \min (0, x)
$$

where we set $y=0.01$ as the negative slope for $x<0$. This prevents the gradient from dying when $x<0$.

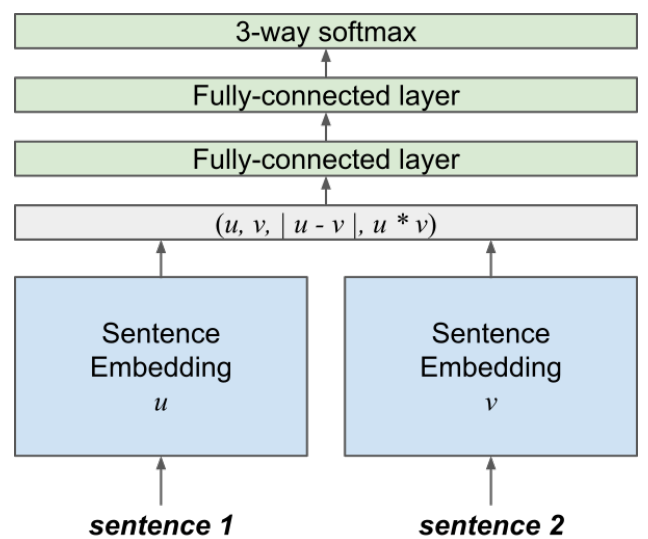

Fig. 1. Overall NLI Architecture

For the sentence representations we first embed the individual words with pretrained word embeddings. The sequence of the embedded words is then passed on to the sentence encoder which utilizes BiLSTM with max pooling. Given a sequence $T$ of words $\left(w_{1} \ldots, w_{T}\right)$, the output of the bi-directional LSTM is a set of vectors $\left(h_{1}, \ldots, h_{T}\right)$, where each $h_{t} \in\left(h_{1}, \ldots, h_{T}\right)$ is the concatenation

$$
h_{t}=\left[\vec{h}_{t}, \overleftarrow{h}_{t}\right]
$$


of a forward and backward LSTMs

$$
\begin{aligned}
\vec{h}_{t} & =\overrightarrow{\operatorname{LSTM}}_{t}\left(w_{1}, \ldots, w_{t}\right) \\
\overleftarrow{h}_{t} & =\overleftarrow{\operatorname{LSTM}}_{t}\left(w_{T}, \ldots, w_{t}\right)
\end{aligned}
$$

The max pooling layer produces a vector of the same dimensionality as $h_{t}$, returning, for each dimension, its maximum value over the hidden units $\left(h_{1}, \ldots, h_{T}\right)$.

Motivated by the strong results of the BiLSTM max pooling network by Conneau et al. (2017), we experimented with combining BiLSTM max pooling networks in a hierarchy-like structure 1 To improve the BiLSTM layers' ability to remember the input words, we let each layer of the network re-read the input embeddings instead of stacking the layers in a strict hierarchical model. In this way, our model acts as an iterative refinement architecture that reconsiders the input in each layer while being informed by the previous layer through initialisation. This creates a hierarchy of refinement layers and each of them contributes to the NLI classification by max pooling the hidden states. In the following we refer to that architecture with the abbreviation HBMP. Max pooling is defined in the standard way of taking the highest value over each dimension of the hidden states and the final sentence embedding is the concatenation of those vectors coming from each BiLSTM layer. The overall architecture is illustrated in Figure 2 .

To summarize the differences between our model and traditional stacked BiLSTM architectures we can list the following three main aspects:

1. Each layer in our model is a separate BiLSTM initialized with the hidden and cell states of the previous layer.

2. Each layer in our model receives the same word embeddings as its input.

3. The final sentence representation is the concatenation of the max pooled output of each layer in the encoder network.

In order to study the effect of our architecture we conduct a comparison of HBMP with the following alternative models:

1. BiLSTM-Ens: Ensemble of three BiLSTMs with max pooling, all getting the same embeddings as the input.

2. BiLSTM-Ens-Train: Ensemble of three BiLSTMs with max pooling, with the hidden and cell states of each BiLSTM being trainable parameters of the whole network.

3. BiLSTM-Ens-Tied: Ensemble of three BiLSTMs with max pooling, where the weights of the BiLSTMs are tied.

4. BiLSTM-Stack: A strictly hierarchical model with three BiLSTM layers where the second and third layer receive the output of the previous layer as their input.

In the first model (BiLSTM-Ens) we contrast our architecture with a similar

\footnotetext{
1 Conneau et al. (2017) explore a similar architecture using convolutional neural networks, called Hierarchical ConvNet.
} 


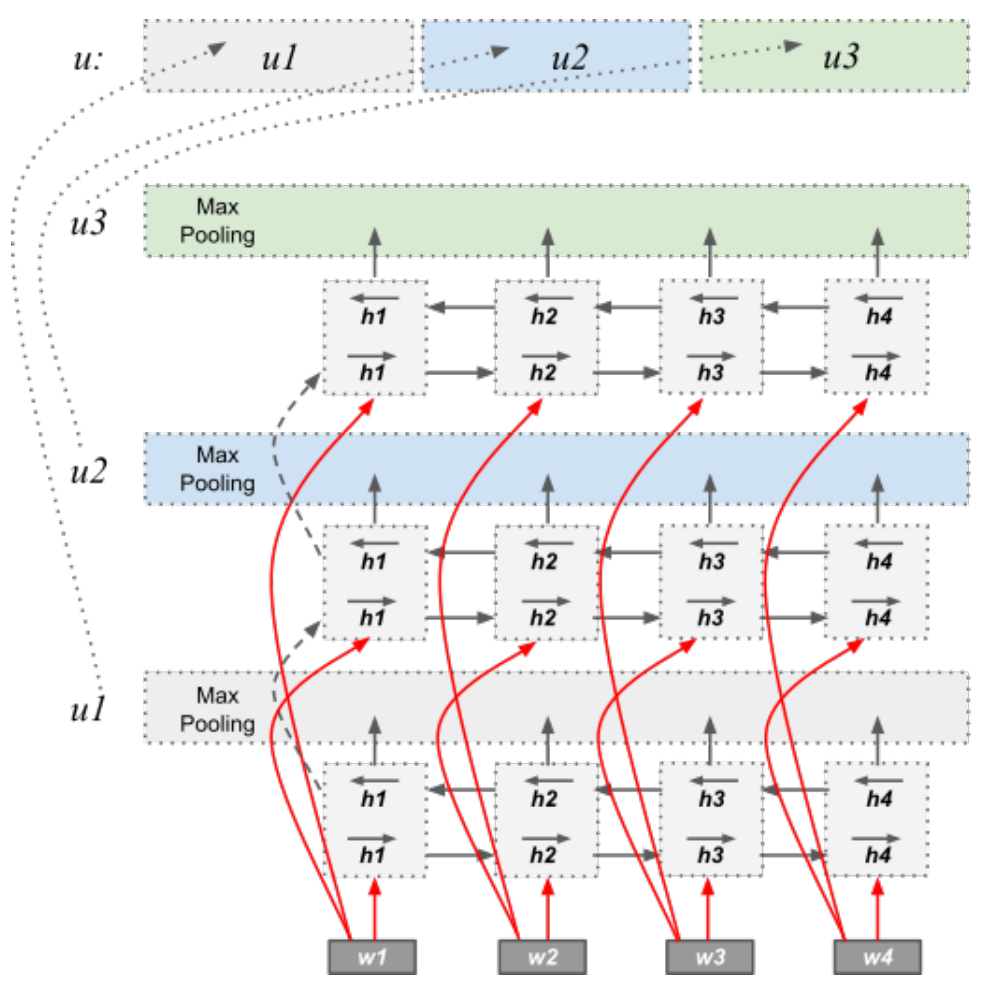

Fig. 2. Architecture of the HBMP sentence encoder (where $T=4$ ).

setup that does not transfer knowledge between layers but also combines information from three separate BiLSTM layers for the final classification. The second model (BiLSTM-Ens-Train) adds a trainable initialization to each layer to study the impact of the hierarchical initialization that we propose in our architecture. The third model (BiLSTM-Ens-Tied) connects the three layers by tying parameters to each other. Finally, the fourth model (BiLSTM-Stack) implements a standard hierarchical network with stacked layers that do not re-read the original input.

We apply the standard SNLI data for the comparison of these different architectures (see Section 5 for more information about the SNLI benchmark). Table 1 lists the results of the experiment.

\begin{tabular}{lcc}
\hline Model & Accuracy & Confidence Interval (95\%)* \\
\hline 600D HBMP (our model) & $\mathbf{8 6 . 6}$ & {$[84.6 \%, 88.7 \%]$} \\
600D BiLSTM-Ens & 86.3 & {$[84.4 \%, 88.3 \%]$} \\
600D BiLSTM-Ens-Train & 86.3 & {$[84.3 \%, 88.4 \%]$} \\
600D BiLSTM-Ens-Tied & 86.1 & {$[83.8 \%, 87.9 \%]$} \\
600D BiLSTM-Stack & 86.3 & {$[84.2 \%, 88.3 \%]$} \\
\hline
\end{tabular}

Table 1. SNLI test accuracies (\%) of different architectures. *Confidence intervals calculated over 1000 random samples of 1000 sentence pairs.

The results show that HBMP performs better than each of the other models, 
which supports the use of our setup in favor of alternative architectures. Furthermore, we can see that the different components all contribute to the final score. Ensembling information from three separate BiLSTM layers (with independent parameters) improves the performance as we can see in the comparison between BiLSTM-Ens and BiLSTM-Ens-Tied. Trainable initialization does not seem to add to the model's capacity and indicates that the hierarchical initialization that we propose is indeed beneficial. Finally, feeding the same input embeddings to all BiLSTMs of HBMP leads to an improvement over the stacked model that does not re-read the input information.

Using these initial findings, we will now look at a more detailed analyses of the performance of HBMP on various datasets and tasks. But before, we first give some more details about the implementation of the model and the training procedures we use. Note, that the same specifications also apply to the experiments that we already discussed above.

\section{Training Details}

The architecture was implemented using PyTorch. We have published our code in GitHub: https://github.com/Helsinki-NLP/HBMP.

For all of our models we used a gradient descent optimization algorithm based on the Adam update rule (Kingma and $\mathrm{Ba}$ 2015), which is pre-implemented in PyTorch. We used a learning rate of 5e-4 for all our models. The learning rate was decreased by the factor of 0.2 after each epoch if the model did not improve. We used a batch size of 64 . The models were evaluated with the development data after each epoch and training was stopped if the development loss increased for more than 3 epochs. The model with the highest development accuracy was selected for testing.

We use pre-trained GloVe word embeddings of size 300 dimensions (GloVe 840B 300D; Pennington et al. 2014), which were fine-tuned during training. The sentence embeddings have hidden size of 600 for both direction (except for SentEval test, where we test models with 600D and 1200D per direction) and the 3-layer multilayer perceptron (MLP) have the size of 600 dimensions. We use a dropout of 0.1 between the MLP layers (except just before the final layer). Our models were trained using one NVIDIA Tesla P100 GPU.

\section{Evaluation Benchmarks}

To further study the performance of HBMP, we train our architecture with three common NLI datasets:

- the Stanford Natural Language Inference (SNLI) corpus,

- the Multi-Genre Natural Language Inference (MultiNLI) corpus,

- the Textual Entailment Dataset from Science Question Answering (SciTail).

Note that we treat them as separate tasks and do not mix any of the training, development and test data in our NLI experiments. We further perform additional linguistic error analyses using the MultiNLI Annotation Dataset and the Breaking 
$N L I$ dataset. Finally, in order to test the ability of the model to learn generalpurpose representations, we apply the downstream tasks that are bundled in the SentEval package for sentence embedding evaluation. Note that we combine SNLI and MultiNLI data in those experiments in order to be compatible with related work. Below we provide a few more details about each of the evaluation frameworks.

SNLI: The Stanford Natural Language Inference (SNLI) corpus (Bowman et al. 2015 ) is a dataset of 570k human-written sentence pairs manually labeled with the gold labels entailment, contradiction, and neutral. The dataset is divided into training (550,152 pairs), development (10,000 pairs) and test sets (10,000 pairs). The source for the premise sentences in SNLI were image captions taken from the Flickr30k corpus (Young, Lai, Hodosh, and Hockenmaier 2014).

MultiNLI: The Multi-Genre Natural Language Inference (MultiNLI) corpus (Williams et al. 2018) is a broad-coverage corpus for natural language inference, consisting of $433 \mathrm{k}$ human-written sentence pairs labeled with entailment, contradiction and neutral. Unlike the SNLI corpus, which draws the premise sentence from image captions, MultiNLI consists of sentence pairs from ten distinct genres of both written and spoken English. The dataset is divided into training $(392,702$ pairs), development (20,000 pairs) and test sets (20,000 pairs).

Only five genres are included in the training set. The development and test sets have been divided into matched and mismatched, where the former includes only sentences from the same genres as the training data, and the latter includes sentences from the remaining genres not present in the training data.

In addition to the training, development and test sets, MultiNLI provides a smaller annotation dataset, which contains approximately 1000 sentence pairs annotated with linguistic properties of the sentences and is split between the matched and mismatched datasets. ${ }^{2}$ This dataset provides a simple way to assess what kind of sentence pairs an NLI system is able to predict correctly and where it makes errors. We use the annotation dataset to perform linguistic error analysis of our model and compare the results to results obtained with InferSent. For our experiment with the annotation dataset we use the annotations for the MultiNLI mismatched dataset.

SciTail: SciTail (Khot et al. 2018) is an NLI dataset created from multiple-choice science exams consisting of $27 \mathrm{k}$ sentence pairs. Each question and the correct answer choice have been converted into an assertive statement to form the hypothesis. The dataset is divided into training (23,596 pairs), development (1,304 pairs) and test sets (2,126 pairs). Unlike the SNLI and MultiNLI datasets, SciTail uses only two labels: entailment and neutral.

2 The annotated dataset and description of the annotations are available at http://www. nyu.edu/projects/bowman/multinli/multinli_1.0_annotations.zip 
Breaking NLI: Breaking NLI (Glockner, Shwartz, and Goldberg 2018) is a test set $(8,193$ pairs) which is constructed by taking premises from the SNLI training set and constructing several hypotheses from them by changing at most one word within the premise. It was constructed to highlight how poorly current neural network models for NLI can handle lexical meaning.

SentEval: SentEval (Conneau et al. 2017, Conneau and Kiela 2018) is a library for evaluating the quality of sentence embeddings ${ }^{3}$ It contains 17 downstream tasks as well as 10 probing tasks. The downstream datasets included in the tests were MR movie reviews, CR product reviews, SUBJ subjectivity status, MPQA opinion-polarity, SST binary sentiment analysis, TREC question-type classification, MRPC paraphrase detection, SICK-Relatedness (SICK-R) semantic textual similarity, SICK-Entailment (SICK-E) natural language inference and STS14 semantic textual similarity. The probing tasks evaluate how well the sentence encodings are able to capture the following linguistic properties: Length prediction, Word Content analysis, Tree depth prediction, Top Constituents prediction, Word order analysis, Verb tense prediction, Subject number prediction, Object number prediction, Semantic odd man out and Coordination Inversion.

For the SentEval tasks we trained our model on NLI data consisting of the concatenation of the SNLI and MultiNLI training sets consisting of 942,854 sentence pairs in total. This allows us to compare our results to the InferSent results which were obtained using a model trained on the same data (Conneau et al. 2017). Conneau et al. (2017) have shown that including all the training data from SNLI and MultiNLI improves significantly the model performance on transfer learning tasks, compared to training the model only on SNLI data.

\section{Model Performance on the NLI task}

In this section, we discuss the performance of the proposed sentence-encoding approach in common natural language inference benchmarks. From the experiments, we can conclude that the model provides strong results on all of the three NLI datasets. It clearly outperforms the similar but non-hierarchical BiLSTM models reported in the literature and fares well in comparison to other state of the art architectures in the sentence encoding category. In particular, our results are close to the current state of the art on SNLI in this category and strong on both, the matched and mismatched test sets of MultiNLI. Finally, on SciTail, we achieve the new state of the art with an accuracy of $86.0 \%$.

Below, we provide additional details on our results for each of the benchmarks. We compare our model only with other state-of-the-art sentence encoding models and exclude cross-sentence attention models, except for SciTail where previous sentence encoding model-based results have not been published.

3 The SentEval test suite is available online at https://github.com/facebookresearch/ SentEval 


\section{$6.1 S N L I$}

For the SNLI dataset, our model provides the test accuracy of $86.6 \%$ after 4 epochs of training. The comparison of our results with the previous state of the art and selected other sentence embedding based results are reported in Table 2 .

\begin{tabular}{lc}
\hline Model & Accuracy \\
\hline BiLSTM Max Pool (InferSent) $^{\mathrm{a}}$ & 84.5 \\
Distance-based Self-Attention $^{\mathrm{b}}$ & 86.3 \\
ReSA $^{\mathrm{c}}$ & 86.3 \\
600D BiLSTM with generalized pooling & $\mathrm{b}$ \\
600D Dynamic Self-Attention Model & 86.6 \\
2400D Multiple-Dynamic Self-Attention Model $^{\mathrm{c}}$ & 86.8 \\
\hline 600D HBMP (our model) & $\mathbf{8 7 . 4}$ \\
\hline
\end{tabular}

Table 2. SNLI test accuracies (\%). Results marked with ${ }^{a}$ by Conneau et al. (2017), ${ }^{\mathrm{b}}$ by Chen, Ling, and Zhu (2018) and ${ }^{\mathrm{c}}$ by Yoon, Lee, and Lee (2018).

\subsection{MultiNLI}

For the MultiNLI matched test set (MultiNLI-m) our model achieves a test accuracy of $73.7 \%$ after 3 epochs of training, which is $0.8 \%$ points lower than the state of the art $74.5 \%$ by Nie and Bansal (2017). For the mismatched test set (MultiNLI-mm) our model achieves a test accuracy of $73.0 \%$ after 3 epochs of training, which is $0.6 \%$ points lower than the state of the art $73.6 \%$ by Chen, Zhu, Ling, Wei, Jiang, and Inkpen (2017b).

A comparison of our results with the previous state of the art and selected other approaches are reported in Table 3 .

Although we did not achieve state of the art results for the MultiNLI dataset, we believe that a systematic study of different BiLSTM max pooling structures could reveal an architecture providing the needed improvement.

\subsection{SciTail}

On the SciTail dataset we compared our model also against non-sentence embedding-based models, as no results have been previously published which are based on independent sentence embeddings. We obtain a score of $86.0 \%$ after 4 epochs of training, which is $+2.7 \%$ points absolute improvement on the previous published state of the art by Tay et al. (2018). Our model also outperforms InferSent which achieves an accuracy of $85.1 \%$ in our experiments. The comparison of our results with the previous state of the art results are reported in Table 4

The results achieved by our proposed model are significantly higher than the previously published results. It has been argued that the lexical similarity of the sentences in SciTail sentence pairs make it a particularly difficult dataset (Khot et al. 2018). If this is the case, we hypothesize that our model is indeed better 


\begin{tabular}{lcc}
\hline Model & $\begin{array}{c}\text { Accuracy } \\
\text { (MultiNLI-m) }\end{array}$ & $\begin{array}{c}\text { Accuracy } \\
\text { (MultiNLI-mm) }\end{array}$ \\
\hline CBOW $^{\mathrm{a}}$ & 66.2 & 64.6 \\
BiLSTM $^{\mathrm{a}}$ & 67.5 & 67.1 \\
BiLSTM + enh embed + max pooling $^{\mathrm{b}}$ & 70.7 & 70.8 \\
BiLSTM + Inner-attention $^{\mathrm{c}}$ & 72.1 & 72.1 \\
Deep Gated Attn. BiLSTM encoders $^{\mathrm{d}}$ & 73.5 & $\mathbf{7 3 . 6}$ \\
Shortcut-Stacked BiLSTM $^{\mathrm{e}}$ & $\mathbf{7 4 . 5}$ & 73.5 \\
\hline 600D HBMP & 73.7 & 73.0 \\
\hline
\end{tabular}

Table 3. MultiNLI test accuracies (\%). Results marked with ${ }^{a}$ are baseline results by Williams et al. (2018), ${ }^{\text {b }}$ by $\mathrm{Vu}(2017),{ }^{\text {c }}$ by Balazs, Marrese-Taylor, Loyola, and Matsuo (2017), ${ }^{\mathrm{d}}$ by Chen et al. (2017b) and ${ }^{\mathrm{e}}$ by Nie and Bansal (2017). Our results for the MultiNLI test sets were obtained by submitting the predictions to the respective Kaggle competitions.

\begin{tabular}{lc}
\hline Model & Accuracy \\
\hline DecompAtt $^{\mathrm{a}}$ & 72.3 \\
ESIM $^{\mathrm{a}}$ & 70.6 \\
Ngram $^{\mathrm{a}}$ & 70.6 \\
DGEM w/o edges $^{\mathrm{a}}$ & 70.8 \\
DGEM $^{\mathrm{a}}$ & 77.3 \\
CAFE $^{\mathrm{b}}$ & 83.3 \\
InferSent & 85.1 \\
\hline 600D HBMP & $\mathbf{8 6 . 0}$ \\
\hline
\end{tabular}

Table 4. SciTail test accuracies (\%). Results marked with ${ }^{\mathrm{a}}$ are baseline results reported by Khot et al. (2018) and ${ }^{\mathrm{b}}$ by Tay et al. (2018).

at identifying entailment relations beyond focusing on the lexical similarity of the sentences.

\section{Error Analysis of NLI Predictions}

To better understand what kind of inferential relationships our model is able to identify, we conducted an error analysis for the three datasets. We report the results below.

Table 5 shows the accuracy of predictions per label (in terms of F-scores) for the HBMP model and compares them to the InferSent model. This analysis shows that our model leads to a significant improvement over the outcome of the nonhierarchical model from previous work in almost all categories on all the three benchmarks. The only exception is the entailment score on SciTail, which is slightly below the performance of InferSent.

To see in more detail how our HBMP model is able to classify sentence pairs with different labels and what kind of errors it makes, we summarize error statistics as confusion matrices for the different datasets. They highlight the HBMP model's strong performance across all the labels. 


\begin{tabular}{l|cc|cc|cc|cc}
\hline & \multicolumn{2}{|c|}{ SNLI } & \multicolumn{2}{c|}{ MultiNLI-m } & \multicolumn{2}{c}{ MultiNLI-mm } & \multicolumn{2}{c}{ SciTail } \\
& нвмP & InferSent & HвмP & InferSent & нвмP & InferSent & HвMP & InferSent \\
\hline entailment & $\mathbf{8 8 . 5}$ & 86.8 & $\mathbf{7 7 . 2}$ & 74.4 & $\mathbf{7 7 . 9}$ & 74.9 & 81.0 & $\mathbf{8 1 . 3}$ \\
contradiction & $\mathbf{8 9 . 1}$ & 86.2 & $\mathbf{7 5 . 3}$ & 71.8 & $\mathbf{7 5 . 6}$ & 71.5 & - & - \\
neutral & $\mathbf{8 1 . 9}$ & 80.9 & $\mathbf{6 8 . 2}$ & 67.1 & $\mathbf{6 8 . 6}$ & 65.4 & $\mathbf{8 8 . 9}$ & 88.1 \\
\hline
\end{tabular}

Table 5. Model performance by F-score, comparing HBMP to InferSent (Conneau et al. 2017) (our implementation).

\begin{tabular}{|c|c|c|c|c|c|c|c|c|}
\hline \multirow{5}{*}{ 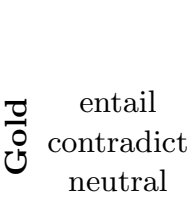 } & \multicolumn{3}{|c|}{ Predicted - HBMP } & \multicolumn{5}{|c|}{ Predicted - InferSent } \\
\hline & entail & contradict & neutral & recall & entail & contradict & neutral & recall \\
\hline & 3047 & 58 & 263 & $90.5 \%$ & 2967 & 95 & 306 & $88.1 \%$ \\
\hline & 117 & 2840 & 280 & $87.7 \%$ & 154 & 2756 & 327 & $85.1 \%$ \\
\hline & 357 & 240 & 2622 & $81.5 \%$ & 346 & 302 & 2571 & $79.9 \%$ \\
\hline precision & $86.5 \%$ & $90.5 \%$ & $82.8 \%$ & & $85.6 \%$ & $87.4 \%$ & $80.2 \%$ & \\
\hline
\end{tabular}

Table 6. SNLI confusion matrices for HBMP and InferSent.

On the SNLI dataset our model clearly outperforms InferSent on all labels in terms of precision and recall. Table 6 contains the confusion matrices for that dataset comparing HBMP to InferSent. The precision on contradiction exceeds $90 \%$ for our model and reaches high recall values for both, entailment and contradiction. The performance is lower for neutral and the confusion of that label with both, contradiction and entailment is higher. However, HBMP still outperforms InferSent by a similar margin as for the other two labels.

Unlike for the SNLI and both of the MultiNLI datasets, on the SciTail dataset our model is most accurate on sentence pairs labeled neutral, having an F-score $88.9 \%$ compared to pairs marked with entailment, where the F-score was $81.0 \%$. InferSent has slightly higher accuracy on entailment, whereas HBMP outperforms InferSent on neutral. Table 7 contains the confusion matrices for the SciTail dataset comparing the HBMP to InferSent. This analysis reveals that our model mainly suffers in recall on entailment detection whereas it performs well for neutral with respect to recall. It is difficult to say what the reason might be for the mismatch between the two systems but the overall performance of our architecture suggests that it is superior to the InferSent model even though the balance between precision and recall on individual labels is different.

The error analysis of the MultiNLI dataset is not standard as it cannot be based on test data. As the labeled test data is not openly available for MultiNLI, we analyzed the error statistics for this dataset based on the development data.

For the matched dataset (MultiNLI-m) our model had a development accuracy of $74.1 \%$. For MultiNLI-m our model has the best accuracy on sentence pairs labeled with entailment, having an F-score of $77.2 \%$. The model is also almost as accurate in predicting contradictions, with an F-score of $75.3 \%$. Similar to SNLI, our model is less effective on sentence pairs labeled with neutral, having an F-score of $68.2 \%$ but, again, the HBMP model outperforms the InferSent on all the labels. Table 8 contains the confusion matrices for the MultiNLI matched dataset comparing the 


\begin{tabular}{|c|c|c|c|c|c|c|}
\hline & \multicolumn{2}{|c|}{ HBMP } & \multicolumn{4}{|c|}{ InferSent } \\
\hline & entail & neutral & recall & entail & neutral & recall \\
\hline & 632 & 210 & $75.0 \%$ & 673 & 169 & $79.9 \%$ \\
\hline & 88 & 1196 & $93.1 \%$ & 140 & 1144 & $89.1 \%$ \\
\hline & $88.0 \%$ & $85.0 \%$ & & $82.8 \%$ & $87.1 \%$ & \\
\hline
\end{tabular}

Table 7. SciTail confusion matrices for HBMP and InferSent based on the development set.

\begin{tabular}{|c|c|c|c|c|c|c|c|c|}
\hline & \multicolumn{3}{|c|}{ Predicted - HBMP } & \multicolumn{5}{|c|}{ Predicted - InferSent } \\
\hline & entail & & neutral & recall & entail & contradict & neutral & recall \\
\hline & 2781 & 196 & 486 & $80.3 \%$ & 2614 & 278 & 587 & $75.1 \%$ \\
\hline & 372 & 2354 & 514 & $72.7 \%$ & 449 & 2241 & 523 & 69. \\
\hline & 528 & 443 & 2158 & $69.0 \%$ & 477 & 507 & 2139 & $68.5 \%$ \\
\hline & $75.6 \%$ & $78.7 \%$ & $68.3 \%$ & & $73.8 \%$ & $74.1 \%$ & $65.8 \%$ & \\
\hline
\end{tabular}

Table 8. MultiNLI-matched confusion matrices for HBMP and InferSent based on the development set.

HBMP to InferSent. Our model improves upon InferSent in all values of precision and recall, in some cases by a wide margin.

For the MultiNLI mismatched dataset (MultiNLI-mm) our model had a development accuracy of $73.7 \%$. or MultiNLI-mm our model has very similar performance as with the MultiNLI-m dataset, having the best accuracy on sentence pars labeled with entailment, having an F-score of $77.9 \%$. The model is also almost as accurate in predicting contradictions, with an F-score of $75.6 \%$. Our model is less effective on sentence pairs labeled with neutral, having an F-score of $68.6 \%$. Table 9 contains the confusion matrices for the MultiNLI Mismatched dataset comparing the HBMP to InferSent and the picture is similar to the result of the matched dataset. Substantial improvements can be seen again, in particular in the precision of contradiction detection.

\section{Evaluation of Linguistic Abstractions}

The most interesting part of the sentence encoder approach to NLI is the ability of the system to learn generic sentence embeddings that capture abstractions, which can be useful for other downstream tasks as well. In order to understand the capabilities of our model we first look at the type of linguistic reasoning that the NLI system is able to learn using the MultiNLI annotation set and the Breaking NLI test set. Thereafter, we evaluate downstream tasks using the SentEval library to study the use of our NLI-based sentence embeddings in transfer learning.

\subsection{Linguistic Error Analysis of NLI Classifications}

The MultiNLI annotation set makes it possible to conduct a detailed analysis of different linguistic phenomena when predicting inferential relationships. We use this to compare our model to InferSent with respect to the type of linguistic properties 


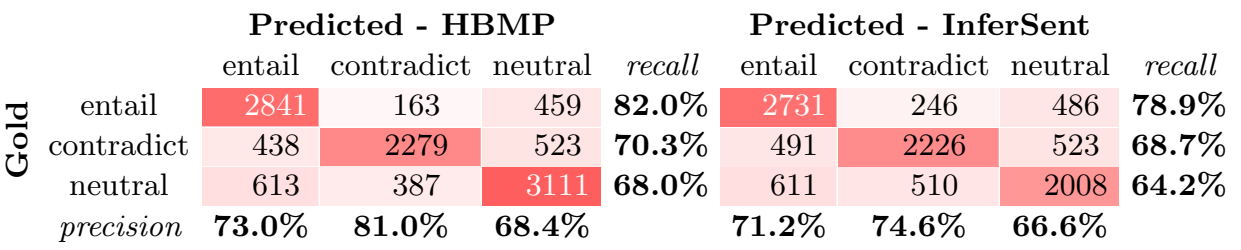

Table 9. MultiNLI-mismatched confusion matrices for HBMP and InferSent.

that are present in the given sentence pairs. Table 10 contains the comparison for the MultiNLI-mm dataset. The analysis shows that our HBMP model outperforms InferSent with antonyms, coreference links, modality, negation, paraphrases and tense differences. It also produces improved scores for most of the other categories in entailment detection. InferSent gains especially with conditionals in contradiction and in the word overlap catehory for entailments. This seems to suggest that InferSent relies a lot on matching words to find entailment and specific constructions indicating contradictions. HBMP does not seem to use word overlap as an indication for entailment that much and is better on detecting neutral sentences in this category. This outcome may indicate that our model works with stronger lexical abstractions than InferSent. However, due to the small number of examples per annotation category and small differences in the scores in general, it is hard to draw reliable conclusions from this experiment.

\begin{tabular}{l|cc|cc|cc}
\hline & \multicolumn{2}{|c|}{ Entailment } & \multicolumn{2}{c|}{ Contradiction } & \multicolumn{2}{c}{ Neutral } \\
& HBMP & InferSent & HBMP & InferSent & HBMP & InferSent \\
\hline active/passive (10) & 100.0 & 100.0 & 100.0 & 100.0 & - & - \\
anto (16) & - & - & $\mathbf{7 6 . 9}$ & 69.2 & $\mathbf{8 5 . 7}$ & 71.4 \\
belief (44) & $\mathbf{8 8 . 2}$ & 82.4 & $\mathbf{6 6 . 7}$ & 61.1 & 73.9 & $\mathbf{7 8 . 3}$ \\
conditional (16) & 81.8 & 81.8 & 37.5 & $\mathbf{6 2 . 5}$ & 57.1 & $\mathbf{7 1 . 4}$ \\
coref (22) & 75.0 & 75.0 & $\mathbf{7 1 . 4}$ & 64.3 & 81.8 & 81.8 \\
long sentence (77) & $\mathbf{8 0 . 6}$ & 77.4 & 58.3 & $\mathbf{6 1 . 1}$ & $\mathbf{7 3 . 8}$ & 71.4 \\
modal (98) & $\mathbf{8 0 . 9}$ & 78.7 & 68.6 & 68.6 & $\mathbf{8 1 . 8}$ & 70.5 \\
negation (78) & $\mathbf{7 6 . 0}$ & 64.0 & $\mathbf{8 1 . 8}$ & 76.4 & $\mathbf{5 8 . 3}$ & 45.8 \\
paraphrase (33) & $\mathbf{8 9 . 2}$ & 86.5 & - & - & & - \\
quantifier (104) & $\mathbf{7 5 . 0}$ & 72.5 & 73.1 & 73.1 & 75.0 & $\mathbf{7 7 . 1}$ \\
quantity/time (15) & 33.3 & $\mathbf{5 0 . 0}$ & 41.7 & 41.7 & 33.3 & 33.3 \\
tense difference (14) & $\mathbf{1 0 0 . 0}$ & 75.0 & 0.0 & 0.0 & $\mathbf{8 3 . 3}$ & 75.0 \\
word overlap (26) & 90.5 & $\mathbf{9 5 . 2}$ & 41.7 & 41.7 & $\mathbf{5 0 . 0}$ & 0.0 \\
\hline Total & 80.6 & $\mathbf{8 3 . 0}$ & 66.3 & $\mathbf{6 8 . 5}$ & $\mathbf{7 3 . 8}$ & 72.7 \\
\hline
\end{tabular}

Table 10. MultiNLI-mm linguistic error analysis (accuracy \%), comparing our HBMP results to the InferSent Conneau et al. (2017) results (our implementation). Number of sentence pairs with the linguistic label in brackets after the label name. 


\subsection{Tests with the Breaking NLI dataset}

In the second experiment we conducted testing of the proposed sentence embedding architecture using the Breaking NLI test set recently published by Glockner et al. (2018). The test set is designed to highlight the lack of lexical reasoning capability of NLI systems.

For the Breaking NLI experiment, we trained our HBMP model and the InferSent model using the SNLI training data. We compare our results with the results published by Glockner et al. (2018) and to results obtained with InferSent sentence encoder (our implementation).

The results show that our HBMP model outperforms the InferSent model in 7 out of 14 categories, receiving an overall score of $65.1 \%$ (InferSent: 65.6\%). Our model is especially strong with handling antonyms, which shows a good level of semantic abstraction on the lexical level. InferSent fares well in narrow categories like drinks, instruments and planets, which may indicate a problem of overfitting to prominent examples in the training data. The strong result on the synonyms class may also come from a significant representation of related examples in training. However, more detailed investigations are necessary to verify this hypothesis.

Our model also compares well against the other models, outperforming Decomposable Attention model (51.90\%) (Parikh, Täckström, Das, and Uszkoreit 2016) and Residual Encoders (62.20\%) (Nie and Bansal 2017) in the overall score. As these models are not based purely on sentence embeddings, the obtained result highlights that sentence embedding approaches can be competitive when handling inferences requiring lexical information. The results of the comparison are summarized in Table 11 .

\subsection{Transfer Learning}

In this section, we focus on transfer learning experiments that apply sentence embeddings trained on NLI to other downstream tasks. In order to better understand how well the sentence encoding model generalizes to different tasks, we conducted various tests implemented in the SentEval sentence embedding evaluation library (Conneau et al. 2017) and compared our results to the results published for InferSent and SkipThought (Kiros et al. 2015).

We used the SentEval library with the default settings recommended on their website, with a logistic regression classifier, Adam optimizer with learning rate of 0.001 , batch size of 64 and epoch size of 4 . Table 12 lists the transfer learning results for our models with 600D and 1200D hidden dimensionality and compares it to the InferSent and SkipThought scores reported by Conneau et al. (2017). Our 1200D model outperforms the InferSent model on 7 out of 10 tasks. The model achieves higher score on 8 out of 9 tasks reported for SkipThought, having equal score on the SUBJ dataset. No MRPC results have been reported for SkipThought.

To study in more detail the linguistic properties of our proposed model, we also ran the recently published SentEval probing tasks (Conneau et al. 2018). Our 1200D 
Sentence Embeddings in NLI with Iterative Refinement Encoders

\begin{tabular}{lcccc|cc}
\hline Category & $\begin{array}{c}\text { Decomp. } \\
\text { Attn }^{*}\end{array}$ & ESIM $^{*}$ & $\begin{array}{c}\text { WordNet } \\
\text { Baseline }^{*}\end{array}$ & KIM $^{*}$ & Senfer- & 600D \\
HBMP
\end{tabular}

Table 11. Breaking NLI scores (accuracy \%). Results marked with * as reported by Glockner et al. (2018). InferSent results obtained with our implementation using the training set-up described in (Conneau et al.|2017). Scores highlighted with bold are top scores when comparing the InferSent and our HBMP model.

\begin{tabular}{l|cc|cc}
\hline Task & InferSent & SkipThought & 600D HBMP & 1200D HBMP \\
\hline MR & 81.1 & 79.4 & 81.5 & $\mathbf{8 1 . 7}$ \\
CR & 86.3 & 83.1 & 86.4 & $\mathbf{8 7 . 0}$ \\
SUBJ & 92.4 & $\mathbf{9 3 . 7}$ & 92.7 & $\mathbf{9 3 . 7}$ \\
MPQA & 90.2 & 89.3 & 89.8 & $\mathbf{9 0 . 3}$ \\
SST & $\mathbf{8 4 . 6}$ & 82.9 & 83.6 & 84.0 \\
TREC & 88.2 & 88.4 & 86.4 & $\mathbf{8 8 . 8}$ \\
MRPC & $76.2 / 83.1$ & - & $74.6 / 82.0$ & $\mathbf{7 6 . 7} / \mathbf{8 3 . 4}$ \\
SICK-R & $\mathbf{0 . 8 8 4}$ & 0.858 & 0.876 & 0.876 \\
SICK-E & $\mathbf{8 6 . 3}$ & 79.5 & 85.3 & 84.7 \\
STS14 & $.70 / .67$ & $.44 / .45$ & $.70 / .66$ & $\mathbf{. 7 1 / . 6 8}$ \\
\hline
\end{tabular}

Table 12. Transfer learning test results for the HBMP model on a number of SentEval downstream sentence embedding evaluation tasks. InferSent and SkipThought results as reported by Conneau et al. (2017). To remain consistent with other work using SentEval, we report the accuracies as they are provided by the SentEval library.

model outperforms the InferSent model in 8 out of 10 probing tasks. The results are listed in Table 13 .

Looking at both the downstream and the probing tasks we can observe strong results of our model compared to the InferSent model that already demonstrated good general abstractions on the sentence level according to the original publication by Conneau et al. (2017). Hence, HBMP does not only provide competitive NLI 


\begin{tabular}{l|c|cc}
\hline Task & InferSent & 600D HBMP & 1200D HBMP \\
\hline SentLen & 71.7 & $\mathbf{7 5 . 9}$ & 75.0 \\
WC & $\mathbf{8 7 . 3}$ & 84.1 & 85.3 \\
TreeDepth & 41.6 & 42.9 & $\mathbf{4 3 . 8}$ \\
TopConst & 70.5 & 76.6 & $\mathbf{7 7 . 2}$ \\
BShift & 65.1 & 64.3 & $\mathbf{6 5 . 6}$ \\
Tense & 86.7 & 86.2 & $\mathbf{8 8 . 0}$ \\
SubjNum & 80.7 & 83.7 & $\mathbf{8 7 . 0}$ \\
ObjNum & 80.3 & 79.3 & $\mathbf{8 1 . 8}$ \\
SOMO & $\mathbf{6 2 . 1}$ & 58.9 & 59.0 \\
CoordInv & 66.8 & 68.5 & $\mathbf{7 0 . 8}$ \\
\hline
\end{tabular}

Table 13. SentEval probing task results (accuracy \%). InferSent results are BiLSTM Max (NLI) results as reported by Conneau et al. (2018).

scores but also produces improved sentence embeddings that are useful for other tasks.

\section{Conclusion}

In this paper we have introduced an iterative refinement architecture (HBMP) based on BiLSTM layers with max pooling that achieves a new state of the art for SciTail and strong results in the SNLI and MultiNLI sentence-encoding category. We carefully analyzed the performance of our model with respect to the label categories and the errors it produces in the various NLI benchmarks. We demonstrate that our model outperforms InferSent in nearly all cases with substantially reduced confusion between classes of inferential relationships. The linguistic analysis on MultiNLI also reveals that our approach is robust across the various categories and outperforms InferSent on, for example, antonyms and negations that require a good level of semantic abstraction.

Furthermore, we tested our model using the SentEval sentence embedding evaluation library, showing that it achieves great generalization capability. The model outperforms InferSent on 7 out of 10 downstream and 8 out of 10 probing tasks, and SkipThought on 8 out of 9 downstream tasks. Overall, our model performs well across all the conducted experiments, which highlights its applicability for various NLP tasks and further demonstrates the general abstractions that it is able to pick up from the NLI training data.

Although the neural network approaches to NLI have been hugely successful, there has also been a number of concerns raised about the quality of current NLI datasets. Gururangan, Swayamdipta, Levy, Schwartz, Bowman, and Smith (2018) and Poliak, Naradowsky, Haldar, Rudinger, and Van Durme (2018) show that datasets like SNLI and MultiNLI contain annotation artifacts which help neural network models in classification, allowing decisions only based on the hypothesis sentences as their input. On a theoretical and methodological level, there is an ongoing discussion on the nature of various NLI datasets, as well as the definition of what counts as NLI and what does not. For example, Chatzikyriakidis, Cooper, 
Dobnik, and Larsson (2017) present an overview of the most standard datasets for NLI and show that the definitions of inference in each of them are actually quite different. Talman and Chatzikyriakidis (2019) further highlight this by testing different state-of-the-art neural network models by training them on one dataset and then testing on another, leading to a significant drop in performance for all models.

In addition to the concerns related to the quality of NLI datasets, the success of the proposed architecture raises a number of other interesting questions. First of all, it would be important to understand what kind of semantic information the different layers are able to capture and how they differ from each other. Secondly, we would like to ask whether other architecture configurations could lead to even stronger results in NLI and other downstream tasks. A third question is concerned with other languages and cross-lingual settings. Does the result carry over to multilingual setups and applications? The final question is whether NLI-based sentence embeddings could successfully be combined with other supervised and also unsupervised ways of learning sentence-level representations. We will look at all those questions in our future work.

\section{Acknowledgments}

The work in this paper was supported by the Academy of Finland through project 314062 from the ICT 2023 call on Computation, Machine Learning and Artificial Intelligence, and through projects 270354/273457/313478.

This project has also received funding from the European Research Council (ERC) under the European Union's Horizon 2020 research and innovation programme (grant agreement No 771113).

We would also like to acknowledge NVIDIA and their GPU grant.

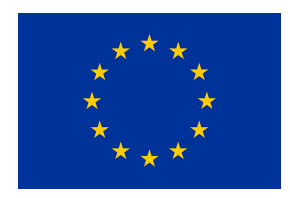

\section{References}

Balazs, J., E. Marrese-Taylor, P. Loyola, and Y. Matsuo 2017. Refining raw sentence representations for textual entailment recognition via attention. In Workshop on Evaluating Vector Space Representations for NLP. ACL.

Bowman, S. R., G. Angeli, C. Potts, and C. D. Manning 2015. A large annotated corpus for learning natural language inference. In EMNLP.

Bowman, S. R., J. Gauthier, A. Rastogi, R. Gupta, C. D. Manning, and C. Potts 2016. A fast unified model for parsing and sentence understanding. In $A C L$.

Chatzikyriakidis, S., R. Cooper, S. Dobnik, and S. Larsson 2017. An overview of natural language inference data collection: The way forward? In Computing Natural Language Inference Workshop.

Chen, Q., Z.-H. Ling, and X. Zhu 2018. Enhancing Sentence Embedding with Generalized Pooling. In COLING.

Chen, Q., X. Zhu, Z.-H. Ling, S. Wei, H. Jiang, and D. Inkpen 2017a. Enhanced lstm for natural language inference. In $A C L$.

Chen, Q., X. Zhu, Z.-H. Ling, S. Wei, H. Jiang, and D. Inkpen 2017b. Recurrent neural network-based sentence encoder with gated attention for natural language inference. In Workshop on Evaluating Vector Space Representations for NLP. ACL. 
Conneau, A. and D. Kiela 2018, May 7-12. SentEval: An evaluation toolkit for universal sentence representations. In N. Calzolari (Ed.), LREC 2018, Eleventh International Conference on Language Resources and Evaluation, Phoenix Seagaia Conference Center, Miyazaki, Japan, pp. 1699-1704.

Conneau, A., D. Kiela, H. Schwenk, L. Barrault, and A. Bordes 2017. Supervised learning of universal sentence representations from natural language inference data. In EMNLP.

Conneau, A., G. Kruszewski, G. Lample, L. Barrault, and M. Baroni 2018. What you can cram into a single vector: Probing sentence embeddings for linguistic properties. In $A C L$.

Glockner, M., V. Shwartz, and Y. Goldberg 2018. Breaking nli systems with sentences that require simple lexical inferences. In $A C L$.

Gururangan, S., S. Swayamdipta, O. Levy, R. Schwartz, S. Bowman, and N. A. Smith 2018. Annotation artifacts in natural language inference data. In NAACL. ACL.

Hill, F., K. Cho, and A. Korhonen 2016. Learning distributed representations of sentences from unlabelled data. In $N A A C L$.

Khot, T., A. Sabharwal, and P. Clark 2018. Scitail: A textual entailment dataset from science question answering. In $A A A I$.

Kingma, D. P. and J. Ba 2015. Adam: A method for stochastic optimization. In ICLR.

Kiros, R., Y. Zhu, R. Salakhutdinov, R. S. Zemel, R. Urtasun, A. Torralba, and S. Fidler 2015. Skip-thought vectors. In NeurIPS.

Maas, A. L., A. Y. Hannun, and A. Y. Ng 2013. Rectifier nonlinearities improve neural network acoustic models. In Intl. Conf. on Machine Learning.

Mikolov, T., I. Sutskever, K. Chen, G. Corrado, and J. Dean 2013. Distributed representations of words and phrases and their compositionality. In NeurIPS, USA.

Mou, L., R. Men, G. Li, Y. Xu, L. Zhang, R. Yan, and Z. Jin 2016. Natural language inference by tree-based convolution and heuristic matching. In $A C L$.

Nie, Y. and M. Bansal 2017. Shortcut-stacked sentence encoders for multi-domain inference. In Workshop on Evaluating Vector Space Representations for NLP. ACL.

Parikh, A. P., O. Täckström, D. Das, and J. Uszkoreit 2016. A decomposable attention model for natural language inference. In $E M N L P$.

Pennington, J., R. Socher, and C. D. Manning 2014. Glove: Global vectors for word representation. In EMNLP.

Poliak, A., J. Naradowsky, A. Haldar, R. Rudinger, and B. Van Durme 2018. Hypothesis only baselines in natural language inference. In Joint Conference on Lexical and Computational Semantics. ACL.

Talman, A. and S. Chatzikyriakidis 2019. Testing the generalization power of neural network models across nli benchmarks. In Proceedings of the 2019 ACL Workshop BlackboxNLP: Analyzing and Interpreting Neural Networks for NLP.

Tay, Y., L. A. Tuan, and S. C. Hui 2018. Compare, compress and propagate: Enhancing neural architectures with alignment factorization for natural language inference. In EMNLP.

Vendrov, I., R. Kiros, S. Fidler, and R. Urtasun 2016. Order-embeddings of images and language.

Vu, H. 2017. Lct-malta's submission to repeval 2017 shared task. In Workshop on Evaluating Vector Space Representations for NLP. ACL.

Williams, A., N. Nangia, and S. R. Bowman 2018. A broad-coverage challenge corpus for sentence understanding through inference. In $N A A C L$.

Yoon, D., D. Lee, and S. Lee 2018. Dynamic Self-Attention : Computing Attention over Words Dynamically for Sentence Embedding. arXiv:1808.07383.

Young, P., A. Lai, M. Hodosh, and J. Hockenmaier 2014. From image descriptions to visual denotations: New similarity metrics for semantic inference over event descriptions. TACL 2. 